Scientific Visualization, 2019, volume 11, number 3, pages 111 - 125, DOI: 10.26583/sv.11.3.10

\title{
Laser holographic interferometry of short ultraviolet radiation with high power density interaction with condensed metters
}

\author{
A.V. Pavlov ${ }^{1}$, Yu.Yu. Protasov², V.D. Telekh³, T.S. Shchepanuk4 \\ Bauman Moscow State Technical University \\ ${ }^{1}$ ORCID: oooo-0002-6593-634X, alekseipavlov@bmstu.ru \\ 2 ORCID: 0000-0001-9271-8500 \\ 3 ORCID: 0000-0003-3270-2122, telekh@bmstu.ru \\ 4 ORCID: 0000-0001-5574-323X
}

\begin{abstract}
The dynamics and macrostructure of near-surface vapor-plasma fluxes arising from condensed substances evaporation in the field of broadband radiation of the UV-VUV spectrum range are studied using double-exposure laser holographic interferometry and schlieren photography (the Toepler scheme in the light field mode).

The experimental setup was created on the basis of the unique scientific facility "BeamM". Broadband UV and VUV radiation was generated by a plasma-dynamic emitting discharge of an end geometry coaxial erosion-type magnetoplasma compressor with an ablating fluoroplastic plasma-forming insulating sleeve in a gas. To control the emission spectrum of the discharge, gas filters were used (Ar, Ne, air, Ne mixture with $5 \%$ air). The emitter operated in the following mode: $\mathrm{U}=20 \mathrm{kV}$, the stored energy $\mathrm{W}=3.6 \mathrm{~kJ}$, the energy input to the discharge was up to $3.2 \mathrm{~kJ}$, with $30 \%$ in the first half-cycle of current, the maximum current is $160 \mathrm{kA}$, the half-cycle of discharge is $6 \mu \mathrm{s}$; the total radiation energy from the discharge was 1.0-1.2 kJ; the fraction of radiation in the VUV range of the spectrum was about $50 \%$ in $\mathrm{Ne}$ (hv $\leq 20 \mathrm{eV}$ ) and about 45\% in $\mathrm{Ar}(\mathrm{hv} \leq 16 \mathrm{eV})$.

It was shown that the implemented scheme of electromagnetic compatibility and protection ensures reliable synchronization of the electric discharge circuit and optical diagnostics components in the whole range of discharge characteristics.

Optical diagnostics of near-surface gas-plasma flows was implemented on the basis of a second harmonic (532 $\mathrm{nm}$ ) of a solid-state Nd:YAG laser (Solar LQ-115), its versatility was shown for studying various pulsed plasma processes with characteristic changes in gas con-

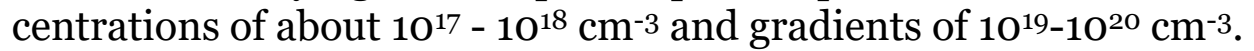

On the schlieren-pictures and interferograms, the zones characteristic of the studied type of radiation effects on materials were recorded: the gas-dynamic evaporation mode was realized (plasma piston mode), there was a shock wave in the gas, the contact boundary between the shock-compressed gas and the vapor plasma. The analysis of interferograms indicates that the regime of developed evaporation was preceded by a regime of diffusion evaporation. The use of extended samples allowed us to observe successive stages in the development of gas-dynamic perturbations during evaporation (due to the different distances between the target areas and the discharge). On the interferograms above the target, at different distances from the source of irradiation, 3 types of gas-dynamic perturbations were observed: acoustic wave, simple wave (Riemann wave), and shock wave.

The effect of the energy characteristics of the discharge and the spectral composition of the radiation on the evaporation pattern was experimentally shown.

Keywords: Keywords: light erosion, double-exposure laser holographic interferometry, light field mode of Toepler's schlieren scheme, dynamics and macrostructure of the nearsurface gas-plasma flows, light radiation of the UV - VUV spectral range.
\end{abstract}




\section{Introduction}

Light erosion gas-plasma flows are generated under high-intensity optical effects on the surface of condensed media. The study of such flows seems relevant both for solving problems of modern physics of a non-equilibrium spatially inhomogeneous plasma, and in connection with numerous technical problems, for example: developing new methods for generating and accelerating gas-plasma flows in plasma-laser accelerators and injectors [1-5], optimization of operating modes of plasma accelerators and electrodynamic devices where the erosion of an insulator and electrodes under the action of radiation determines the mass flow rate of the plasma-forming substance [6-9], process optimization in technological (photolithography, radiation surface hardening) and photochemical installations [10-13]. The interaction of powerful broadband radiation with matter accompanies the processes on the walls of thermonuclear facilities $[14,15]$. This explains the interest in these studies.

A photoerosion gas-plasma flow expanding into the external environment - as a diagnostic object, is rather complex and differs in a wide range of parameters - medium-mass velocities $\tilde{\mathrm{v}} \sim 10^{4}-10^{6} \mathrm{~cm} / \mathrm{c}$, densities $n \sim 10^{15}-10^{19} \mathrm{~cm}^{-3}$, temperatures $T \sim 0,5-50 \mathrm{kK}$, complex macrostructure, chemical and ionization composition. In this case, it is possible to realize high pressure and temperature gradients [3, 7] and solid-gas-plasma phase transitions in intense light fields $I_{0} \sim 10^{5}-10^{9} \mathrm{~W} / \mathrm{cm}^{2}$ and shock-wave loads $\left(M_{i} \sim 0,3-3, p_{1}>10^{6} \mathrm{~Pa}\right)$ [16]. In addition, the study of the dynamics and macrostructure of light erosion gas-plasma flows is complicated by the need to visualize large optical fields.

This requires the use of joint quantitative and qualitative research methods, the development of a complex diagnostic technology of the experiment. Complementary optical methods allow us to obtain quantitative information about the refractive indices and density of the gas-plasma medium, and under our conditions implemented as a synthesis of interferometric, schlieren, and direct-flow methods, which makes it possible to obtain - density fields, density gradient, second derivative of the density of the medium, respectively [17-19].

\section{Experimental conditions}

The dynamics and macrostructure of near-surface vapor-plasma flows arising from evaporation of condensed substances (CV) in the field of broadband radiation of the UV-VUV spectral range were explored.

Targets were made of $\mathrm{Al}, \mathrm{Cu}, \mathrm{Ti}, \mathrm{Pb}, \mathrm{C},\left(\mathrm{C}_{2} \mathrm{~F}_{4}\right)_{\mathrm{n}}$. in the form of bars with dimensions of 30 $\mathrm{mm}$ by $50 \mathrm{~mm}$ and a thickness of $10 \mathrm{~mm}$ and set the long side along the discharge at a distance of $45 \mathrm{~mm}$ from the axis of the PDD (Fig. 1). Practically, the PTFE installed below has always been used as a reference target, in order to exclude contamination of the studied samples by the products of its ablation.

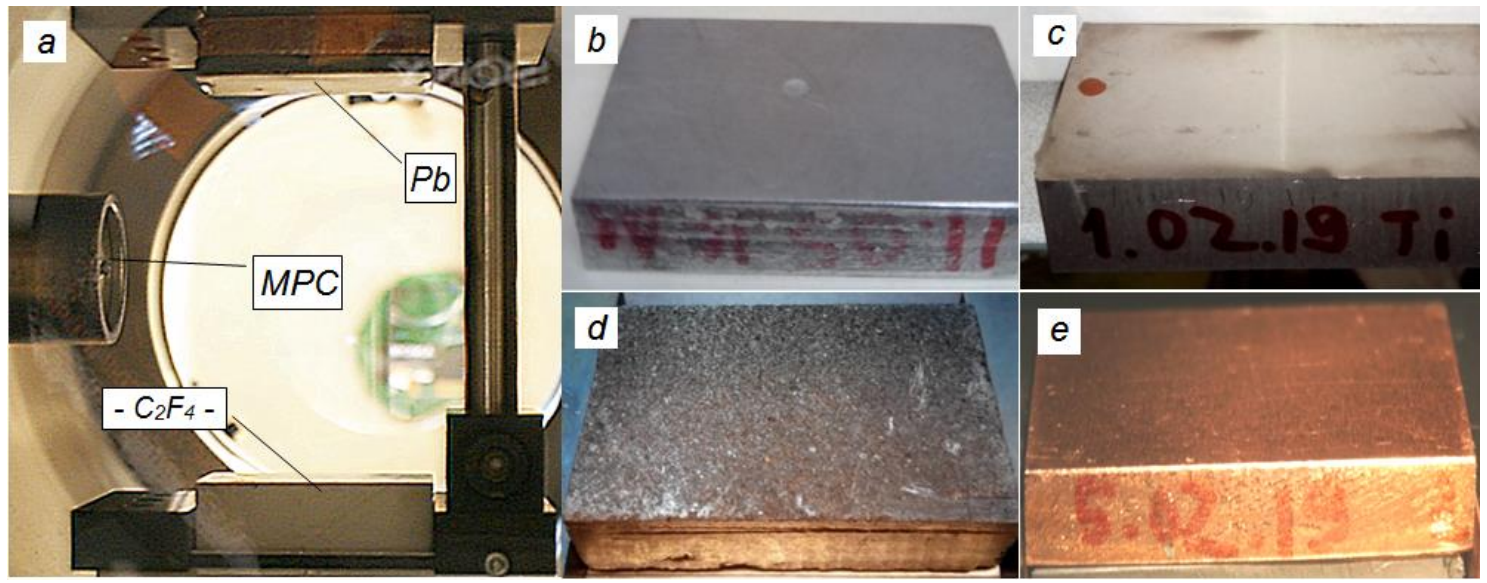

Fig.1. Setting the target in the discharge chamber (a) and the type of target $\mathrm{Al}-(\mathrm{b}), \mathrm{Ti}-(\mathrm{c}), \mathrm{C}-(\mathrm{d}), \mathrm{Cu}-(\mathrm{e})$ 
As a radiation source, a plasmodynamic radiating discharge (PDD) in a gas (Ne, $\mathrm{Ar}$, Air, Neon with the $5 \%$ addition of Air; $\mathrm{P}_{\mathrm{Ar}}=200$ Torr, $\mathrm{P}_{\mathrm{Ne}}=400$ Torr) was used, formed in a pulsed electromagnetic plasma accelerator of erosion type with electrodes of a coaxially end geometry (diameter 6 and $34 \mathrm{~mm}$ ) and an ablating PTFE plasma-forming sleeve that is described in $[16,20]$.

Such discharges are characterized by high spectral radiation brightness characteristics in the near UV and vacuum UV (VUV) regions of the spectrum and have an extended glow body. According to the conventional concepts [7] the structure of these discharges consists of the radiating plasma zone gasdynamically unperturbed gaseous medium and lying between a layer of shock-compressed gas. If such a discharge occurs in dense gases, then high-speed (30-6o km / s) quasi-stationary plasma flow of erosion products of the separation dielectric ablative bushes. This stream of gas is inhibited on the deformable barrier, it's kinetic energy thermalized in the shock wave and partially radiated. Luminous efficiency of such types of devices is $40-60 \%$ of the energy that is deposited in the plasma [7, 20].

Shortwave radiation spectrum boundary of bits determined is by the first ionization potential of the background gas. Control emission spectrum PDD of MPC emission in gases is that the gas filters are used to change the spectral range. For this purpose, the background gas (matrix) is highly absorbing additive - gaseous substance with a low ionization potential. The amount of additive selected conditions ensure efficient absorption of the hard radiation is filtered, but should not significantly alter the density and electrical properties of the gaseous medium, and accordingly, change the time and geometric characteristics of the discharge. As background gas is most expedient to use neon, which is characterized by the second highest after helium ionization potential of $21.6 \mathrm{eV}$ and relatively high density under normal conditions $m_{N e}=20$. Since neon has a specific structure energy levels characterized in that the resonant lines are near the first ionization potential, for the shorter transmission boundary neon adopted radiation quanta energy $\mathrm{hn}=20 \mathrm{eV}$.

Radiation properties of the gas discharge in the energy range of photons to $20 \mathrm{eV}$ are described in [16]. The radiation flux density generated by the MPC luminous surface discharge on a target calculated as in [21]. In the first approximation, it is approximated by hemisphere-illuminated zone whose radius increases with time. Act to increase the radius of the hemisphere was determined by a series of Toeplergrams.

For the described radiator's mode $(U=20 \mathrm{kV}$, the stored energy is $W=3,6 \mathrm{~kJ}$ energy deposited in the discharge up to $3.2 \mathrm{~kJ}$, with $30 \%$ - in the first half cycle current, the maximum current of $160 \mathrm{kA}$, the discharge half 6 microseconds) distribution energy over spectral ranges shown in table 1 (for a pure neon discharge) and table 2 (for a neon supplemented with $5 \%$ air).

Table 1

The spectral energy distribution of the radiation source $\mathrm{hn}=0-20 \mathrm{eV}$

\begin{tabular}{|c|c|c|c|c|}
\hline $\begin{array}{l}\text { The energy } \\
\text { range, eV }\end{array}$ & Dhn, eV & $E_{\text {mid, }} \mathrm{J}$ & $\%$ & $E_{\text {mid }} / \mathrm{D} h \mathrm{n}, \mathrm{J} / \mathrm{eV}$ \\
\hline$h \mathrm{n}=\mathrm{O}-20$ & 20 & 1190 & 100 & 59,4 \\
\hline$h \mathrm{n}=0,5-6,2$ & 5,7 & 600 & 50,5 & 105 \\
\hline$h \mathrm{n}=0,5-3,1$ & 2,6 & 333 & 28,1 & 128 \\
\hline$h \mathrm{n}=0,5-11,2$ & 10,7 & 817 & 68,8 & 76,4 \\
\hline$h \mathrm{n}=4,35-5,0$ & 0,65 & 52 & 4,4 & 80 \\
\hline$h \mathrm{n}=2,3-3,16$ & 0,86 & 70 & 5,9 & 81,4 \\
\hline
\end{tabular}


Table 2

The spectral energy distribution of the radiation source $h \mathrm{n}=0-6,7 \mathrm{eV}$ (neon with $5 \%$ air addition)

\begin{tabular}{|l|l|l|l|l|}
\hline $\begin{array}{l}\text { The energy } \\
\text { range, eV }\end{array}$ & Dhn, eV & $E_{\text {mid, }}$ J & $\%$ & $E_{\text {mid }} /$ Dhn, J/eV \\
\hline$h n=0-6,7$ & 6,7 & 995 & 100 & 149 \\
\hline$h n=0,5-6,2$ & 5,7 & 896 & 90 & 157 \\
\hline$h n=0,5-3,1$ & 2,6 & 301 & 30 & 116 \\
\hline$h n=0,5-6,7$ & 6,2 & 822 & 83 & 133 \\
\hline$h n=4,35-5,0$ & 0,86 & 62 & 6 & 72 \\
\hline$h n=2,3-3,16$ & 0,65 & 50 & 5 & 72 \\
\hline
\end{tabular}

The total radiation energy from the discharge reached $1.2-1.6 \mathrm{~kJ}$. The share of radiation in the VUV spectrum (quanta energy hn more than $6 \mathrm{eV}$ ) is $45 \%$ (in $\mathrm{Ar}$, hn to $16 \mathrm{eV}$ ) and about $50 \%$ (in $\mathrm{Ne}, \mathrm{hn}$ to $20 \mathrm{eV}$ ). In the visible region of the spectrum, the time course of the radiation is a trapezium with a leading edge of $3 \mu \mathrm{s}$, a shelf of $8 \mu \mathrm{s}$, and a gentle decay of $20 \mu \mathrm{s}$ duration (see oscillogram, Fig. 4). The density of the radiation flux at the near to discharge end of the target at a maximum is about $P_{\text {vis }}=0,4 \mathrm{MW} / \mathrm{cm}^{2}$ (on the basis that the discharge radiation irradiates the sphere of radius $3 \mathrm{~cm}, 3 \mathrm{~cm}$ radius is the distance from the axis of discharge to the proximal end of the target). In fact, the density of the radiation flux on the target above, since the radiating surface approaching to the target. In the VUV region, the radiation appears in front of the current maximum and its duration is $60-70 \%$ of the half-life of the discharge. If this pulse is approximated by a triangle with a base of $4 \mu$ s and a leading edge of $1.5 \mu \mathrm{s}$ [7] and taking into account that the first half-period accounts for 2/3 of the energy of this range, then we obtain that the radiation flux density in the VUV range at maximum will be Rvuv $=2.5 \mathrm{MW} / \mathrm{cm}^{2}$. Also note that on the near-end of the target to the MPC end edge fell $2-2.5$ times more energy than on the far-end.

\section{Experimental setup}

The experimental setup is realized on the basis of unique scientific installation "Beam-M» (http://ckp-rf.ru/usu/200975, http://usu-beam.bmstu.ru) is shown in Figure 2.

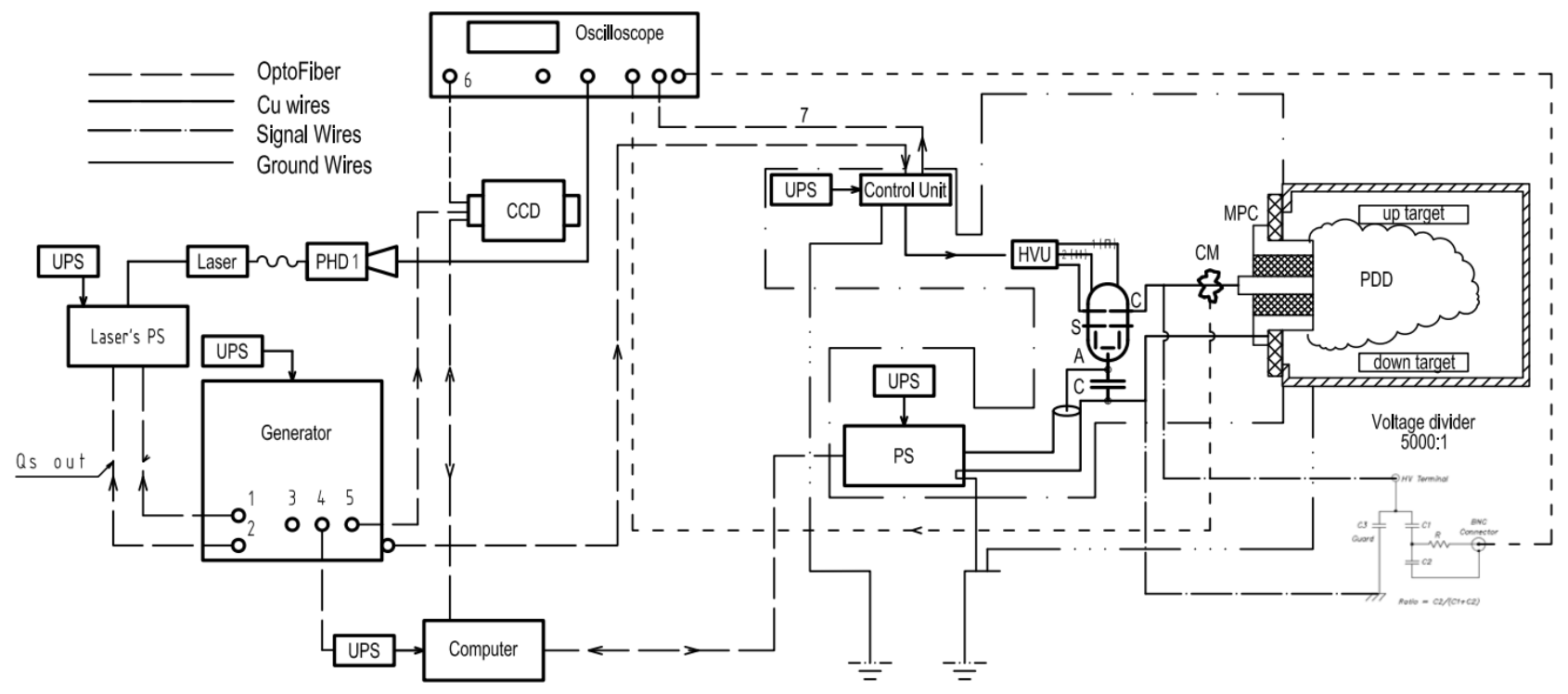

Fig.2. Experimental setup 
Since the radiation source was a high-current pulse discharge, then for EMC measures were taken to discharge shielding assembly (DA) consist of the capacitor, thyratron (both with the high-voltage ignition unit) and MPC generating pulsed electromagnetic interference from the rest of sensitive equipment. To do this, the PC was placed in a grounded Faraday copper grid with a mesh size of approx. $1 \mathrm{~mm}$ and sensitive control equipment in a metal case (figure 2.). All electrical equipment has been isolated by supply chains, both among themselves and from external noise sources. For transmitting control signals to the ignition control unit, the CCD camera and the capacitor-charging unit are optocouplers used. For power, signal and protective ground connection we have used specially ground independently conducted wires those controlled ground impedance is about $2 \mathrm{ohms}$. These measures have provided a stable synchronization for virtually every discharge.

Laser diagnostics subsurface gas-plasma flow resulting from the evaporation of condensed substances powerful broadband radiation comprised of double-exposure holographic interferometry $[19,22]$ and schlieren Toepler's circuit in the light field mode. Advantages of holographic interferometry to classical schemes are as follows. As it is known, in classical interferometers the interference pattern is formed by light waves propagating simultaneously on different channels (arms of the interferometer).This leads to the high quality requirements of the optical elements through which the interfering wave. The holographic interferometry interfere waves transmitted by one and the same path, but at different times and recorded in the same hologram (Method two exposures). When the hologram is illuminated two waves are reduced reference beam corresponding to the two states of the object. When these interference waves formed interferograms fringes shifts which are due only to a change of state of the object between the first and second exposures. All wavefront distortion introduced by the optical parts, almost entirely offset by $[22,23]$. Reduced requirements for quality optics can increase the size of the objects.

Optical diagnostics was carried out according to the Toepler's schlieren scheme in the light field mode and by the method of double-exposure holographic interferometry, which were implemented on the basis of a solid-state Nd:YAG laser (Solar LQ-115, $\mathrm{l}_{2}=532 \mathrm{~nm}$, $E_{\text {pulse }}=23 \mathrm{~mJ}, \mathrm{t}_{\text {pulse }}=7 \mathrm{~ns}$ ). The optical module scheme for the installation is shown in Fig. 3, see also [16].

In the holographic interferometer scheme, mirror 7 divides the laser beam into objective and reference in the 1: 1 ratio in intensity, the final intensity ratio in the arms was selected experimentally using neutral light filters 25 located in front of the hologram. The intrinsic emission of the discharge was cut off by a narrow-band interference filter 21. The optical path lengths with an accuracy of $1 \mathrm{~cm}$ were aligned with a mirror 8 mounted on a graduated rail, which made it easier to reconfigure the circuit for different experiments $[24,25]$. Note that in our case interferograms of large aperture objects are recorded with a diameter of up to 200 $\mathrm{mm}$. The object beam was expanded with a telescope 2 and lenses 12, 13 and after passing through phase inhomogeneity in chamber 3 using a lens system $14-17$ was collected by a parallel beam on screen 6. The reciprocal arrangement of lenses $14-17$ made it possible to get a clear focused image of the object with a practically compensated level of distortion. The reference beam passed through mirrors 8 and 9, leveling the optical paths and, expanding with a telescope from lenses 19-18, hit the hologram.

When implementing the double-exposure holographic interferometry method [22], the first laser pulse was produced before the onset of the process under study; the second - during the discharge with the required delay relative to its beginning. To register interferograms in bands of a finite width (which allows one to obtain absolute values of the change in the refractive index), a quartz wedge with an apex angle of $5^{\circ}$ was inserted into the object beam, which by turning around the vertical axis by an angle of $30-50$ between two exposures changed the angle between the reference and subject rays. The frequency of the bands was chosen taking into account the convenience of decoding interferograms and at given angles of rotation it was possible to change the distance between the bands in the range of $1-0.5 \mathrm{~mm}$. 


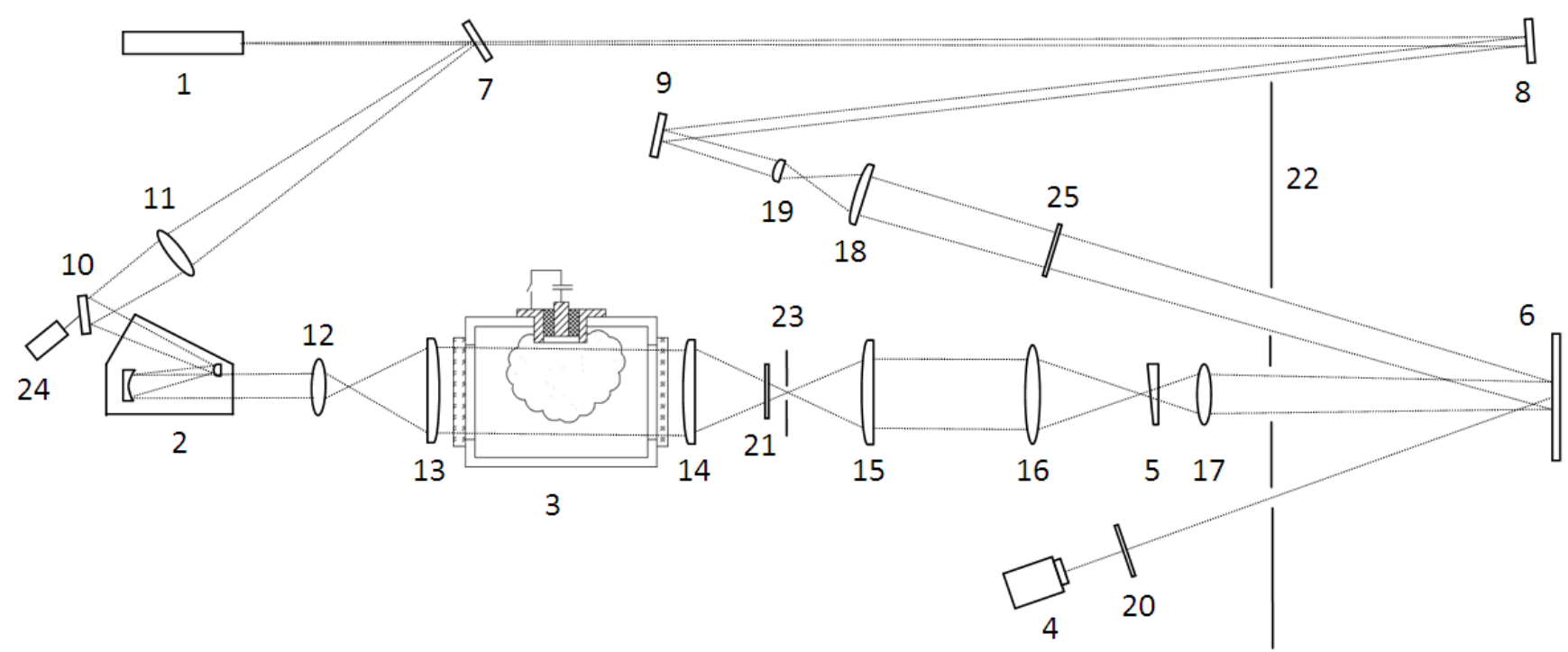

Fig. 3. Optical setup: 1 - $532 \mathrm{~nm}$ laser, 2 - reflector laser beam expander, 3 - vacuum chamber, 4 - CCD camera Videoscan VS-285C, 5 - wedge $5^{\circ}, 6$ - screen and installation location of the film, 7-10 - mirrors, 11-19- lenses, 20 - neutral protective filter, 21 - interference filter $532 \mathrm{~nm}, 22$ - light blocking screen, 23 - diaphragm of $1.2 \mathrm{~mm}, 24$ - photodiode FDUK 2UT, 25 - neutral density filter

Thus, a hologram of a focused image [26] was recorded on a holographic film of a VRP-M sensitive to the green region of the spectrum [where a certain point of the object corresponds to each hologram point), which made it possible to reconstruct holograms with a digital mirror camera in white light of a conventional presentation projector, having the film approximately at the angle of exposure.

To implement the method of schlieren-photography in the scheme of fig. 3 screen 22 overlapped the reference beam and in the focus of the lens 14 with a focal length of $400 \mathrm{~mm}$ was placed a diaphragm 23 with a diameter of $1.2 \mathrm{~mm}$. For the registration of the schlierentenegram in place of screen 6, both a VRP-M film and a white matte screen were installed, which made it possible to use a high-speed video camera for quick selection of parameters and process diagnostics.

The spatial resolution of the diagnostic unit was determined by the resolution of the optical system and the recording system (from $100 \mu \mathrm{m}$ for a digital camera and less than 50 $\mu \mathrm{m}$ for photo film), and the temporal (10 ns) resolution was determined by the laser pulse duration.

The sensitivity of the schlieren method can be estimated by setting the deflection angle of the rays on the optical inhomogeneity $a=1(d n / d x)$ (where $d n / d x$ is the gradient of the refractive index in the perpendicular direction; 1 is the optical path in the phase inhomogeneity, $\mathrm{l}=3 \mathrm{~cm}$ (target's width)) equal to half the angular size of the diaphragm: $\mathrm{a}_{\mathrm{d}}=\mathrm{d} / \mathrm{F}$ (where $\mathrm{d}=$ $1.2 \mathrm{~mm}$ is the diameter of the aperture opening; $\mathrm{F}=400 \mathrm{~mm}$ is the focal length of the lens in front of the diaphragm) and using the ratio [19] $n-1=2 \mathrm{pa}_{\mathrm{a}} N$, which relates the gas concentration to its refractive index (aa is the atomic polarizability, with [27]). Then, the minimum transverse gradient of the gas component concentration recorded by the Schlieren method is $\sim 2 \times 10^{20} \mathrm{~cm}^{-4}$ for neon and $0.5 \times 10^{20} \mathrm{~cm}^{-4}$ for argon.

For interferometry, the minimum recorded change in gas concentration is:

$$
\Delta N_{\min }=\frac{1}{2 \pi \alpha_{a}} \frac{\lambda \Delta k}{l}
$$

where $\Delta k$ - is the shift of the band relative to the unperturbed gas, 1 - is the wavelength of the probe radiation $(532 \mathrm{~nm})$. If we assume that the minimum recorded shift of the band is $\Delta k$ $\min =0,1$, then for neon $\mathrm{D} N_{\min }=0,7 \cdot 10^{18} \mathrm{~cm}^{-3}$, and for $\arg$ on $\mathrm{D} N_{\min }=0,17 \cdot 10^{18} \mathrm{~cm}^{-3}$. 
The instrumental errors and systematic errors can be in experimental conditions, that influence the determination of the function $\mathrm{n}(\mathrm{x})$, associated with the parallelism of the probe laser radiation with the plane of the target surface (see. Fig. 4). If the probe radiation (beam $\mathrm{AB}$ ) makes an angle a with target plane (oZ axis) shift of the fringes as the result of changes in refractive index in the range of the $\mathrm{x}$ coordinate from $\mathrm{x}_{1}$ to $\mathrm{x}_{2}$ (rather than at some fixed, $\mathrm{x}=$ const). Parallelism of the probe beam and the target plane is determined by the width of the flat shock wave front occurring at target. Since the thickness of the front shock wave is several mean free path (i.e., in our conditions, it should have substantially zero width), then measuring the width Dh portion at which the interference fringe is deflected from the unperturbed value to a maximum value of SW, can be determined the degree of parallelism.
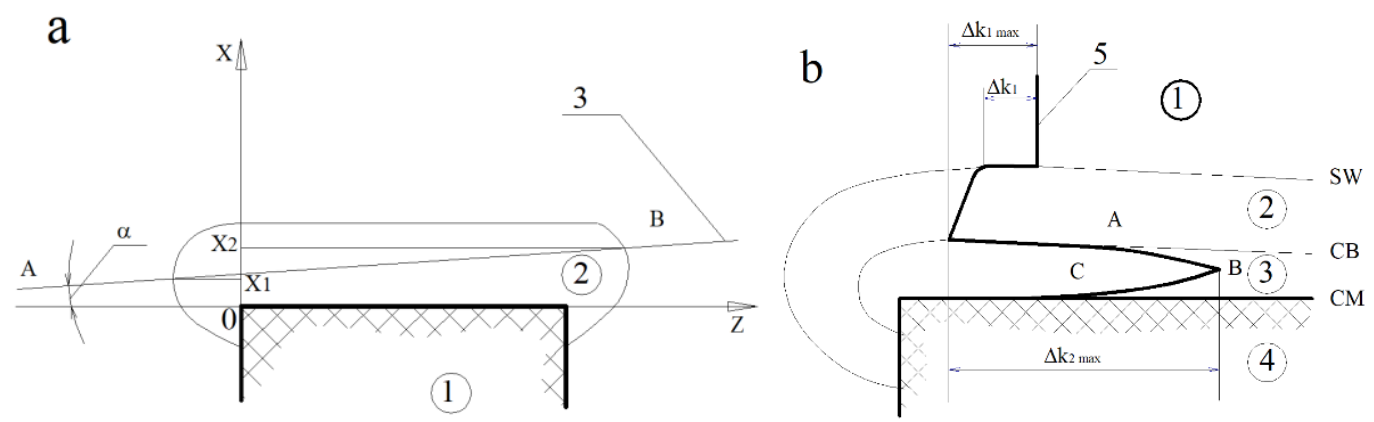

Fig. 4. Determination of methodological errors in sensing phase inhomogeneities: a - diagram for explaining the occurrence of errors in sensing phase heterogeneity: 1 - target; 2 - light erosion plasma; $\mathrm{AB}$ - direction of the probe radiation; $\mathrm{b}$ - shift circuit of the interference fringes in the region RHD - action on the target: 1 - undisturbed gas; 2 - shockcompressed gas; 3 - light erosion plasma; 4 - target; 5 - interference fringe; SW - shock wave; CB - contact boundary; CM - condensed matter

\section{Discussion of The Results}

\section{Schlieren pictures}

Fig.5 is a Schlieren photograph or Toeplergrams of the discharge and the corresponding current waveform, and the voltage signal from the photodetector. It shows the location of the target relative to the discharge. The peak in the signal from the photodetector corresponds to the moment of shooting.
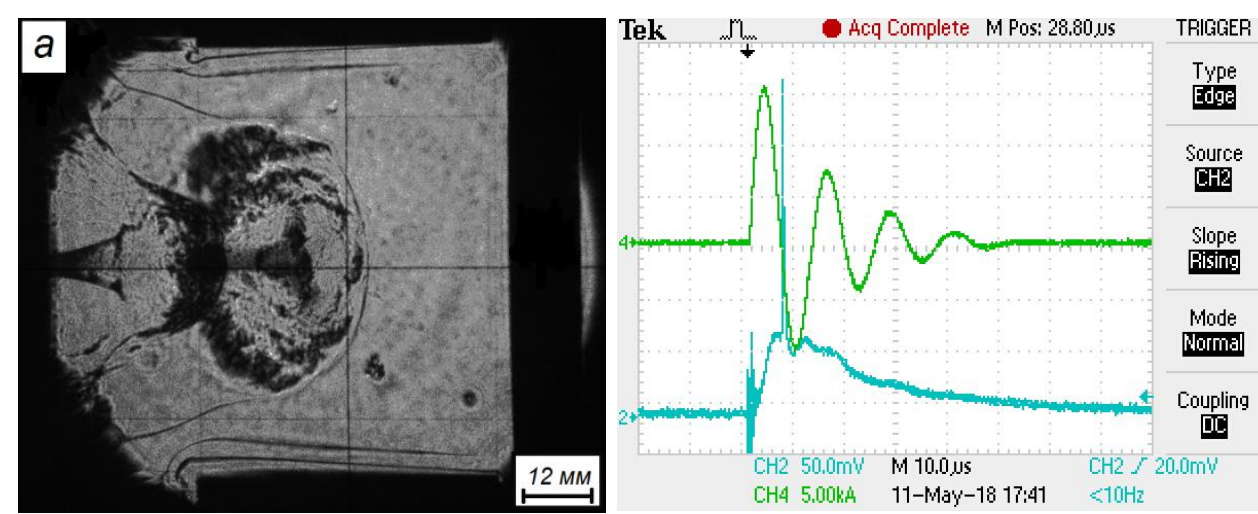

Fig.5. Toeplergram in Neon gas ( $p=400$ Torr, aperture is $1.2 \mathrm{~mm}$ ); Target from the bottom PTFE $50 \times 30 \mathrm{~mm}$, from above - polished copper $50 \times 30 \mathrm{~mm}$ (a) A typical oscillogram of the plasmadynamic discharge: channel 4 - discharge current, channel 2 - signal from the photodiode with laser shooting pik corresponded to Toeplergramm registration moment (b) 
According to schlieren pictures (an example is shown in Fig. 6), it was found that all materials studied evaporate under the action of discharge radiation in neon and argon, and two zones stand out above the target: a shock-compressed gas zone (separated from the background gas by a shock wave (SW)) ) and the target vapor zone (separated from the shockcompressed gas by the contact boundary (CB)) [20]. Thus, the "plasma piston" mode is realized - the target material evaporates, the vapors heat up, ionize and push like a piston surrounding the gas, creating an SW in it.

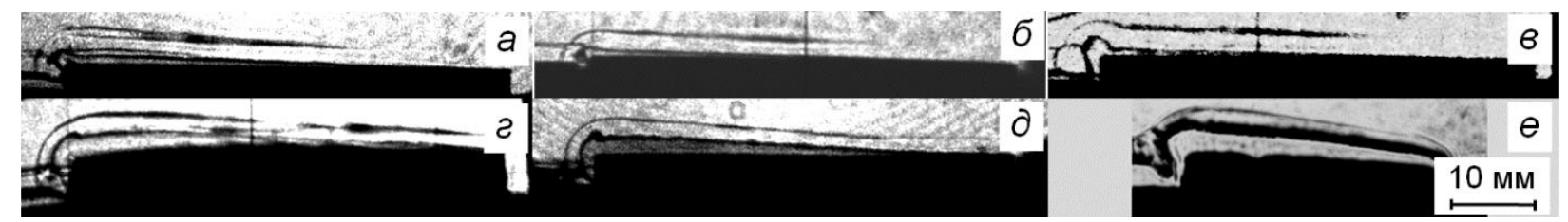

Fig.6. Toeplergrams of light erosion of steam-plasma flows with targets in argon $\left(P_{A r}=200\right.$ Torr, $U_{c}=20 \mathrm{kV}, t=9.6 \mathrm{~ms}(\mathrm{a}, \mathrm{e})$ and $t=12 \mathrm{~ms}(\mathrm{~b}, \mathrm{c}, \mathrm{d}, \mathrm{f})$, aperture is $1.2 \mathrm{~mm}$ (a, d, e, f), 1.5 mm (b), 1.8 mm (c)); a - Al; b - Cu; c - Ti; d - Pb; e - PTFE; f - beech (type of wood)

According to the intensity of evaporation, the target materials can be divided into two groups: easily evaporating (PTFE (photodestruction), graphite (low thermal conductivity), $\mathrm{Pb}$ (low boiling point)) and hardly evaporating ( $\mathrm{Ti}, \mathrm{Cu}, \mathrm{Al}$ ). Also, from the Toeplergrams it can be seen that the state of the target surface (its roughness, which was expected), as well as the presence in the buffer gas of impurities of the ablative fluoroplastic plasma-forming sleeve and the studied targets remaining from the previous discharge. In addition, the occurrence of SW was recorded at a considerable distance from the target surface, despite the fact that the piston (target and CB pairs) was not yet observed (Fig. 6(c)). This is explained by the heating of the target vapor that has diffused into the gas, as will be discussed below.

\section{Holographic interferometry}

Using the scheme (Fig. 3), a series of evaporation interferograms of all the materials studied was obtained at the same time instant $-9.5 \mu \mathrm{s}$ from the start of the discharge. Targets in turn were located in the same place, while the reference target (the "witness target") from the PTFE was the same and did not move.

When a lignt wave passes through plasma stream that is introduced into it, the amplitude value relates to absorption and scattering and phase distortion. Exploring these distortions, it is possible to obtain information on absorption and refractive index, which is a function of temperature and concentration of the components of the steam plasma particle flow.

To a first approximation, in the described scheme in the optical path length of the phase inhomogeneity can be assumed constant (independent of distance from the surface) and a width equal to the target, i.e., most have a single bond shifts bands with a refractive index when an object is homogeneous with respect to the line of sight, i.e. refractive index depends only on the coordinates $x, y$ (two-dimensional distribution) [19]:

from whence

$$
\begin{aligned}
\Delta \varphi(x, y) & =\frac{2 \pi L}{\lambda}\left[n(x, y, z)-n_{0}\right] \text { (phase shift) } \\
K(x, y) & =\frac{L}{\lambda}\left[n(x, y, z)-n_{0}\right] \text { (bands shift), }
\end{aligned}
$$

$$
n(x, y)=K(x, y) \frac{\lambda}{L}+n_{0},
$$

where $L$ - geometric length of the beam path in the inhomogeneity.

The problem of determining changes in refractive index thus reduced to a measurement of the interferogram fringe shift as a function of the coordinates $x, y$. 
The test surface layer is a gas-plasma stream, in which there are electrons, ions and neutral atoms and molecules. The contribution of different kinds of particles in the refraction can be considered additive, and the refractive index is equal to $n-1=\sum_{\mathrm{K}} C_{\mathrm{K}} N_{\mathrm{K}}$, where $C_{\mathrm{K}}$ - refraction particles $K$ th sort per one particle; $N_{\mathrm{K}}$ corresponding number of particles per unit volume. The contribution to the electron and ion (atomic) components give opposite signs. Thus, to move from the measured value of the refractive index to the parameters of plasma is necessary either to use additional assumptions or interferometry to produce at several wavelengths.

Assuming that there is a buffer gas between the SW and $\mathrm{CB}$, the deviation of the interference fringes of the target in the area is proportional to the change in the gas density. For the region between the target and $\mathrm{CB}$ to conduct analysis more complex, since in this region there is a plasma vapor targets and contribute to the change in refractive index is given as ions (with the sign "+") and electrons (with the sign "-")

Figure 7 shows a holographic interferogram of the evaporation of copper target in argon.

As already noted, the targets in our experiments were very long $(50 \mathrm{~mm})$, end the target end closest to the radiation source was 2-2.5 times more energy than the far end. In this case, the deviation of the interference bands was recorded over the entire length of the target, but the shift of the bands differed noticeably when moving from one edge of the target to the other. Above the target, at different distances from the source of radiation (from the PDD) we distinguish three types of gas-dynamic perturbations: an acoustic wave, a simple wave, and a weak shock wave.

At the far (from the PDD) edge of the target (see Fig. 7 b, e, lane 1) an acoustic wave is recorded. Its distinctive features are that there is a disturbance at the leading edge, but behind this front the interference fringe follows without deviation, i.e. pressure and density are almost the same as background gas. The shift of the interference band is less than $\mathrm{D} k \leq 0,1$, which corresponds to a change in the concentration of not more than $\Delta N_{\mathrm{Ar}} \leq 0,17 \cdot 10^{18} \mathrm{~cm}-$ 3. Then the gas density jump is $\Delta N / N_{0}=0,024$ (here $N_{0}=7,1 \cdot 10^{18} \mathrm{~cm}^{-3}-$ is the concentration of argon at a pressure of 200 Torr). A perturbation with such a small density difference spreads with a sound speed $(\mathrm{v}=350 \mathrm{~m} / \mathrm{s}$ for argon at $T=20 \mathrm{C})$. The distance from the disturbance front to the target is $h=2 \mathrm{~mm}$. From these data, you can determine the point in time when this disturbance arose: $t=h / v=6 \mu \mathrm{s}$. 

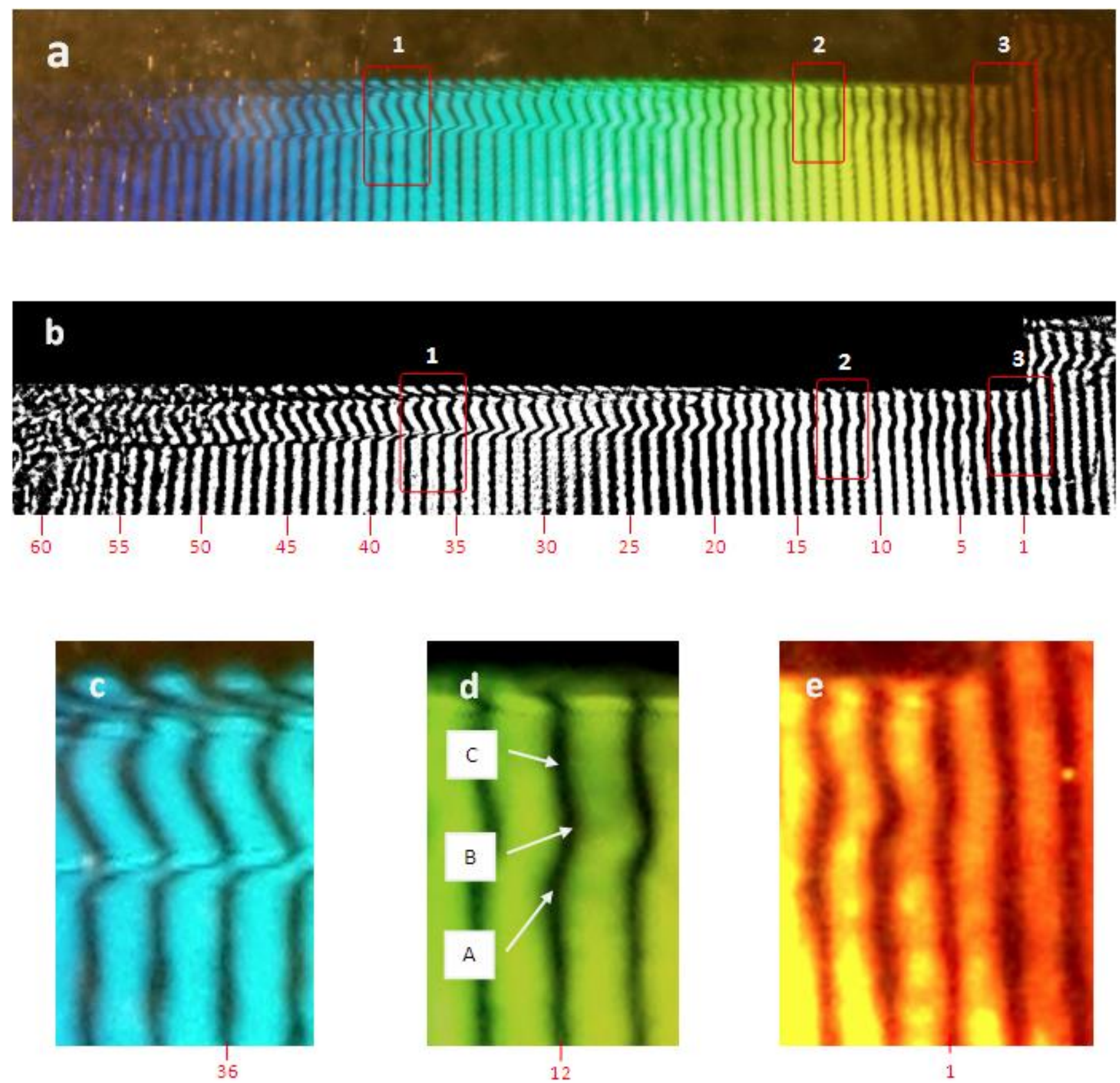

Fig. 7. Holographic interferogram of a copper target in argon evaporation $\left(p_{\mathrm{Ar}}=200\right.$ Torr): a is a hologram reconstructed in white light and recorded by a camera (Sony a7) with selected areas 1, 2, 3, which correspond to characteristic gas-dynamic perturbations (strip spacing of $0.8 \mathrm{~mm}$ ); $\mathrm{b}$ - the same in monochrome mode, $\mathrm{c}$ - enlarged area 1 corresponding to the shock wave, $\mathrm{d}$ - enlarged area 2 corresponding to a simple Riemann wave, e - enlarged area 3 corresponding to acoustic disturbance.

The interference band No. 12 (Fig. 4 b, d) corresponds to a simple wave (Riemann wave) created by the plasma piston moving with acceleration. The density jump at the leading edge is small ( $\mathrm{D} k=0,1-0,2$, и $\Delta N / N_{0}=0,03-0,04-$ is small), and the disturbance front moves with the speed of sound. At the section $A B$, the density increases and a shock wave will be formed here later. After the maximum deviation (point B), the density decreases - the BC section. If the piston moved all the time with the same acceleration, then behind the leading edge, as the piston approaches, the gas density should increase [28]. The decrease in density, in our case, means that after the initial acceleration the piston slows down its movement by the time of receiving the interferogram (perhaps even the acceleration has changed its sign). At the edge of the target, closest to the radiation source, a shock wave was observed - the third type of gas-dynamic perturbation. There have a steep leading edge (Fig. 7 b, c, strip No. 
36). When $\mathrm{D} k=1$ we get $\Delta N_{\mathrm{Ar}}=1,7 \cdot 10^{18} \mathrm{sm}^{-3}$. This density difference $\left(\rho / \rho_{0}=\left(\Delta N_{\mathrm{Ar}}+\right.\right.$ $\left.\left.N_{0}\right) / N_{0}=1,24\right)$ corresponds to a Mach number equal $M=1,15(\gamma=5 / 3)$.

Since the deviation of interference fringes above the target is proportional to the change in the gas density, the gas compression behind the shock wave can be estimated using two methods. For the case shown in Fig. 8 (PTFE, Ar, $x=0.5 \mathrm{~cm}$ from the target edge), the distance from the shock to the surface of the target is 2 times greater than from the SW to the CB. The gas that occupied the SW - the target's surface (TS) interval before discharge - now occupies the SW - CB interval. Thus, the compression ratio here is $\rho / \rho_{o}=2.2$. On the other hand, the shift of the bands at this point is $\Delta \mathrm{k}=4$. This shift corresponds to the compression $\rho / \rho_{o}=\left(\Delta \mathrm{N}_{\mathrm{Ar}}+\mathrm{N}_{\mathrm{o}}\right) / \mathrm{N}_{\mathrm{o}}=2$. It turns out the correspondence between the two methods. We add that the compression $\rho / \rho_{o}=2.2$ corresponds to the Mach number $\mathrm{M}=2$.

The interference pattern (Fig. 7) that the distance from the target surface to the boundary of the perturbation is practically independent of the distance from the radiation source.

Since the speed of these disturbances is approximately the same (the speed of sound in the background gas), they arose at approximately one instant of time from the beginning of the discharge. Moreover, for different materials this moment is approximately the same: $6-7$ microseconds before the image is taken or 3.5-4.5 microseconds after the start of the discharge, which approximately corresponds to the time to reach the first maximum current. Thus, there is an approximately simultaneous occurrence of disturbances along the entire length of the target, while the absorbed energy is significantly different: as noted above, the absorbed energy at the end of the target close to the PDD is 2-2.5 times greater than at the far end.

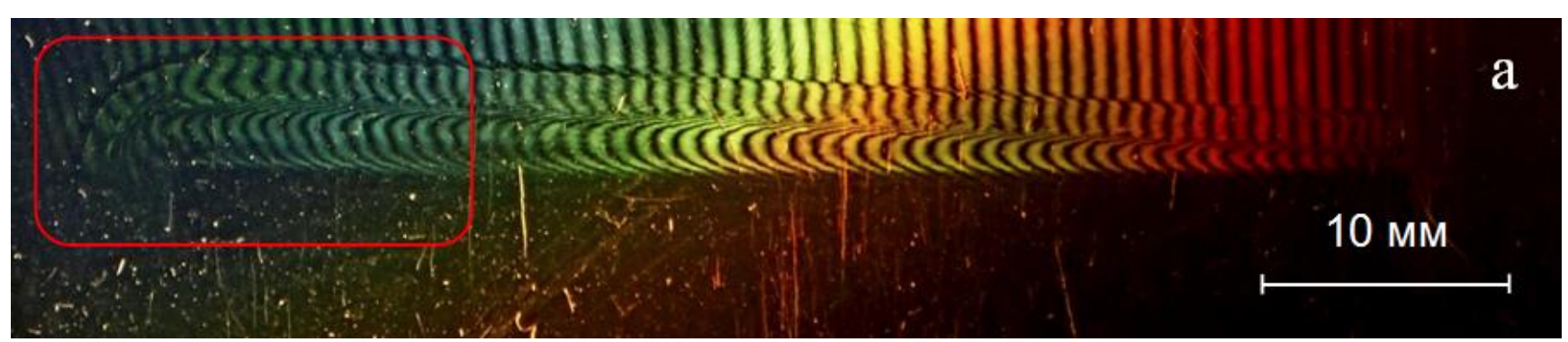

Fig 8. Holographic interferogram of a PTFE target in argon evaporation $\left(p_{\mathrm{Ar}}=200\right.$ Torr $)$

This result has the following explanation. At the first stage, at the beginning of the discharge, a relatively soft radiation heats the surface and the target vapor diffuses into the surrounding gas. During the diffusion of vapors into the gas, an acoustic disturbance does not occur. The second stage begins when a hard radiation with a sharp leading edge comes from the discharge, which corresponds to a moment in time close to the maximum current. Vapors diffused into the gas absorb hard radiation, ionize, heat up and, expanding, cause acoustic disturbance. As can be seen at this point in time, the pairs appeared over the entire surface, but in different quantities, therefore the intensity of the resulting wave is different. Further heating of the surface leads to the fact that the pressure in the vapor reaches the pressure of the surrounding gas, a contact boundary between the vapor and the gas arises, and the developed evaporation regime is realized [29]. The mode when a diffusion mixture of target vapor and ambient gas arises was studied under the influence of laser radiation on matter experimentally [30] and theoretically [31].

To confirm the two-stage evaporation mechanism, two experiments were performed. Firstly, neon with the addition of air (2\% in pressure) was used as a buffer gas, which changed the spectral composition of the radiation that hit the target (in this case there was no hard component, and the quanta energy did not exceed $6 \mathrm{eV}$ ). total energy [20]. In this case, the SW and CBs appeared much later over metal targets, and from a certain distance from the PDD did not even appear at all. At the same time, the evaporation mode of the PTFE "witness 
target" remained almost unchanged. The intense evaporation of the PTFE is explained by photodestruction [32] and the energy of the incident quanta is less than $6 \mathrm{eV}$ for this mechanism.

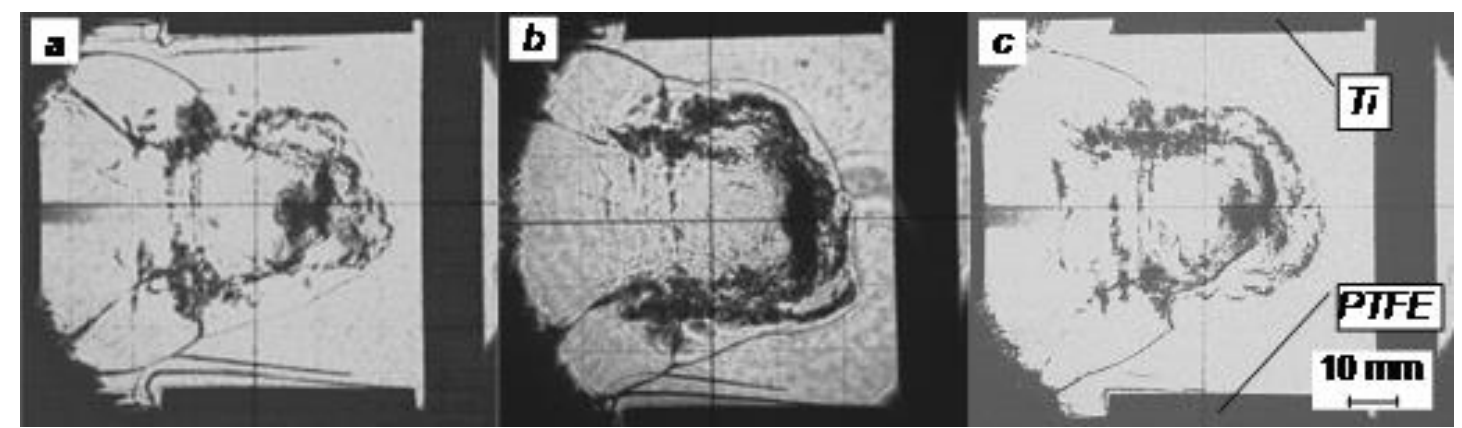

Fig.9. Toeplergrams of light erosion gas-plasma flows in $\operatorname{argon}\left(U_{c}=20 \mathrm{kV}, t=12 \mu \mathrm{s}, P_{A r}=\right.$ 200 Torr (a), $4^{\text {th }}$ discharge (b), added air 20 Torr (c), aperture is $1.8 \mathrm{~mm}(\mathrm{a}), 1.2 \mathrm{~mm}(\mathrm{~b}), 1.1$ $\mathrm{mm}(\mathrm{c}))$.

Secondly, the charging voltage of the capacitor was reduced to $15 \mathrm{kV}$, which reduced the energy input to the discharge by 1.8 times, and as many times less than the light energy hit the target. In this case, the perturbation of the interference bands did not appear along the entire surface of the target, but the front of this perturbation was equidistant from the surface. The calculated moment of occurrence of this disturbance indicates that it appeared at the time of maximum discharge current.
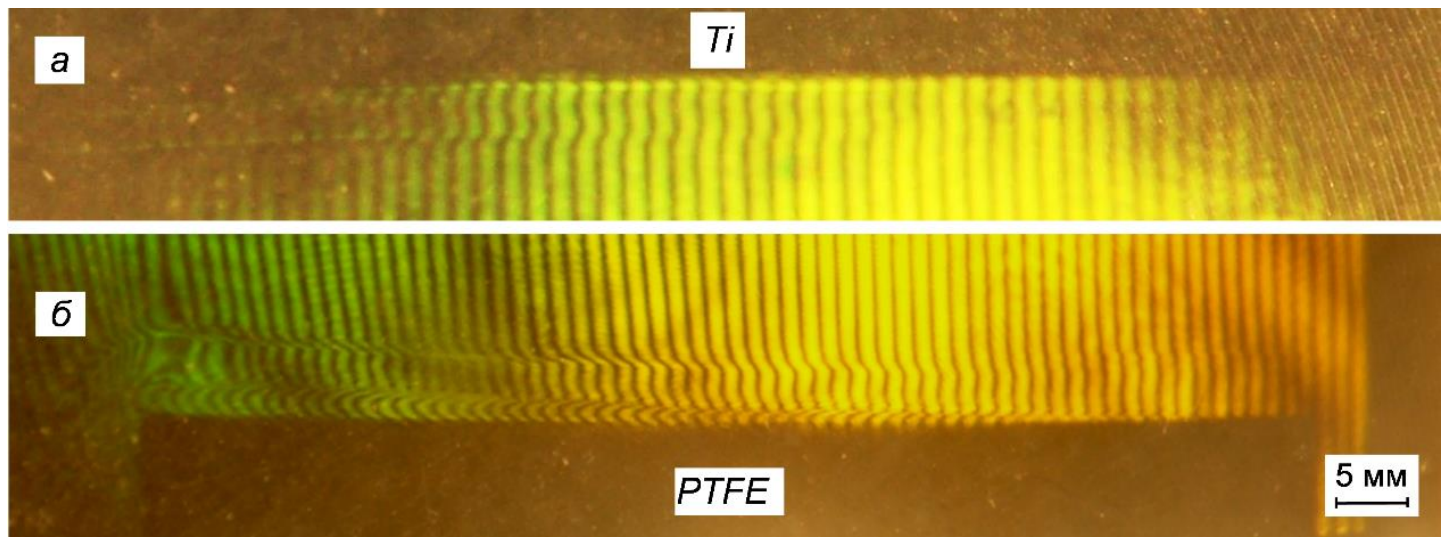

Fig.10. Holographic interferogram of fluoroplastic target's evaporation in $\operatorname{argon}\left(U_{c}=15 \mathrm{kV}, t\right.$ $=10 \mathrm{~ms}, P_{A r}=200$ Torr $)$

\section{Conclusion}

We take advantage of the two-exposure laser holographic interferometry and Toepler schlieren-schemes in the light field mode to investigate the dynamics and macrostructure of near-surface vapor-plasma flows arising from evaporation of condensed substances in the field of broadband radiation of the UV - VUV range of the spectrum.

On the schlieren-pictures and interferograms, the zones characteristic of the studied type of radiation effect on materials are recorded: the gas-dynamic evaporation mode is realized (plasma piston mode), there is a shock wave in the gas, the contact boundary between the shock-compressed gas and the vapor plasma. The analysis of interferograms indicates that the regime of developed evaporation is preceded by a regime of diffusion evaporation. On interferograms above the target at different distances from the source of radiation (from the PDD), we observe 3 types of gas-dynamic perturbations: acoustic wave, simple wave (Riemann wave), and shock wave. 
Research has been performed at "Beam-M" facility, following the government task by the Russian Ministry of Education and Science (13.6918.2017/8.9), partially supported by the Russian Foundation for Basic Research (grant 18-29-21039).

\section{References}

1. Schou J., Amoruso S., Lunney J. / Laser Ablation and its Applications / Ed. C. Phipps. Berlin, Heidelberg:Springer, 2007. P. 67.

2. Loktionov E.Y., Protasov Y.Y. Experimental study of the dynamics and macrostructure of laser-induced high-pressure dust gas-plasma flows // High Temperature. 2011. V. 49, N 1. PP. 36-44.

3. Protasov Yu.S., Protasov Yu.Yu. Laser propulsion: radiative gasdynamic and thermophysical interchamber processes of double-stage laser rocket thruster. I // 36th AIAA/ ASME/ SAE/ ASEE Joint Propulsion Conf.: AIAA paper № 2000-3485.- Huntsville: AIAA, 2000.$9 \mathrm{p}$.

4. Kuzenov V.V., Ryzhkov S.V. // J. Phys.: Conf. Ser. 2017. V. 830. P. 012124

5. Protasov Y. S., Protasov Y. Y., Suslov V. I., Telekh V. D. Experimental research of radiative gasdynamic and thermophysical processes in plasma optical converter of laser radiation into AC electrical current. I. // Proceedings of the 35 Intersociety Energy Conversion Engineering Conference: AIAA paper 2000-2888. -N.Y.: AIAA, 2000. -P. 476-483.

6. Radiative Plasmodynamics vol 1 / ed Yu. S. Protasov. Moscow: Energoatomizdat, 1991.

7. Protasov Yu S 2000 Plasma light sources of high spectral brightness Enciclopedia of Low Temperature Plasma. Introduction voluem. Vol IV ed V E Fortov (Moscow: Nauka) 232

8. Popov G.A., Antropov N.N. Ablative PPT. New quality, new perspectives // Acta Astronautica. 2006. 59. P. 175

9. Popov G.A., Khrustalev M.M., Khrabrov V.A., Antropov N.N., Lyubinskaya N.V. // Plasma Phys. Rep. 2014. V. 40, N 5. P. 336

10. EUV Sources for Lithography / ed. V Bakshi. Wash. Bellingham: SPIE Press, 2006.

11. Chkhalo N.I., Salashchenko N.N. // Nucl. instrum. and methods in phys. research. 2009. A 603 (1-2). P. 147

12. Arkhipov V.P., Kamrukov A.S., Kozlov N.P., Makarchuk A.A. // Applied Physics. 2016. N 6. P. 102

13. Kirillov D.V., et. al. The structure and properties of modified surface carbon steel by compression plasma flow // J. Phys.: Conf. Ser. 2018. V. 1115. P. 032036.

14. Vyacheslavov L.N. et al. // Physica Scripta. 2018. V. 93. P. 035602

15. Kovalenko D.V. et al. // Problems of Atomic Science and Technology, Ser. Thermonuclear Fusion. 2014. V. 37, N 4. P. 39.

16. Pavlov A., Protasov Yu., Telekh V., Tshepanuk T. Experimental Research of Dynamics and Macrostructure of Light Erosion Radiative Plasmodynamic Discharges // J. Phys.: Conf. Ser. 2017. V. 830. P. 012062

17. Vasil'ev L.A. Tenevye metody.- M.: Nauka, 1968.- 480 s.

18. Hariharan P. Optical Interferometry. San Diego: Academic Press, 2003. - 368 p.

19. Zaidel' A.N., Ostrovskaya G.V. Lazernye metody issledovaniya plazmy. L.: Nauka, 1977. $219 \mathrm{c}$.

20. Nosov K.V., et al. Experimental investigation of powerful wide band radiation interaction with the condensed matters // Journal of Physics: Conference Series. 2018. V.1115. P. 032011.

21. Anderzhanov A K, Volokhovich S Yu, Divnov I I and Zotov N I // J. of Appl. Mechanics and Tech. Phys. 1990. V. 2. P. 8

22. Ostrovskii Yu.I. Golografiya i ee primenenie. L.: Nauka, 1973. 180 c.

23. Ostrovskaya G.V. Golographic interferometry of physical processes // Technical Physics, 61(6), 799-814. doi:10.1134/S1063784216060153.

24. Skryabin A.S., et al. // Journal of Physics: Conference Series. 2018. V.1115. P. 042017 
25. Nosov K.V., Pavlov A.V., Telekh V.D., Shchepanyuk T.S. Investigation of parameters plasmodynamic laser-induced discharge in magnetoplasma compressor end geometry // Russian Physics Journal. 2017. V.60, N 10-2. P.78-83.

26. Golograficheskaya interferometriya fazovykh ob"ektov. / pod red. G.I. Mishina, L.: Nauka, 1979. $232 \mathrm{~s}$.

27. Peter Schwerdtfeger, Jeffrey K. Nagle Table of static dipole polarizabilities of the neutral elements in the periodic table // Molecular Physics. 2018. DOI: 10.1080/00268976.2018.1535143

28.Landau L.D., Lifshits E.M. Gidrodinamika. M.: Nauka, 1988.

29. Vorob'ev V.S. Plasma arising during the interaction of laser radiation with solids // Phys. Usp. 36 (12) 1129-1157 (1993).

30.Dan'shchikov E.V., Dymshanov V.A., Lebedev F.V., Ryazanov A.V. Structure and properties of an optical breakdown plasma in a $\mathrm{CO} 2$ laser beam near a target surface // Soviet Journal of Quantum Electronics. 1985. V. 15, N 5, P. 659-663.

31. Borets-Pervak I.Yu., Vorob'ev V.S. Plasma formation threshold in a vapor-gas mixture on the surface of a laser-heated metal // Soviet Journal of Quantum Electronics. 1991. V. 21, N 8. P. 905-908.

32. Loktionov E.Yu., et al. Energy efficiency of femtosecond laser ablation of polymer materials // Journal of Applied Spectroscopy. 2012. V. 79, N 1. P. 104-112. 